\title{
Tumor duodenal del estroma gastrointestinal: una causa poco frecuente de hemorragia digestiva
}

\author{
Armando Baeza-Zapata ${ }^{1}$, Susanna Scharrer-Cabello , Carlos Herrera-Figueroa ${ }^{1}$, Óscar García-de León², \\ Edwars Rodríguez-Hinojosa ${ }^{3}$ y Joel O. Jáquez-Quintana ${ }^{1 *}$ \\ ${ }^{1}$ Departamento de Gastroenterología y Endoscopia Digestiva; ${ }^{2}$ Departamento de Cirugía General; ${ }^{3}$ Departamento de Anatomía Patológica. Hospital \\ Universitario José Eleuterio González, Monterrey, N.L., México
}

\begin{abstract}
Resumen
Los tumores del estroma gastrointestinal (GIST) representan la mayoría de los tumores mesenquimales del tracto gastrointestinal, solo el 5\% se localizan en el duodeno. Los avances en los últimos 30 años en el campo de la medicina molecular, genética e inmunohistoquímica han permitido identificar mejor este subgrupo de tumores y brindar un tratamiento específico. Presentamos el caso de un hombre de 61 años con historia de melena y síndrome anémico en el que se encontró un GIST ulcerado en la tercera porción del duodeno con respuesta favorable al tratamiento médico y quirúrgico.
\end{abstract}

Palabras clave: Tumor del estroma gastrointestinal. Hemorragia gastrointestinal. Tumores duodenales.

\section{Duodenal gastrointestinal stromal tumor: an uncommon cause of digestive tract bleeding}

\begin{abstract}
Gastrointestinal stromal tumors (GIST) constitute most mesenchymal tumors of the gastrointestinal tract, only $5 \%$ can be found in the duodenum. Advances in the last 30 years in the field of molecular medicine, genetics, and immunohistochemistry have enabled to better identify this subgroup of tumors and facilitate specific treatment. We present the case of a 61-year-old male with melena and anemic syndrome in which an ulcerated GIST was found in the third portion of the duodenum with a favorable response to medical and surgical treatment.
\end{abstract}

Key words: Gastrointestinal stromal tumor. Gastrointestinal hemorrhage. Duodenal neoplasms.

\section{Introducción}

Los tumores del estroma gastrointestinal (GIST) son tumores malignos poco frecuentes que pueden presentarse en cualquier parte del tracto gastrointestinal ${ }^{1}$, se estima una incidencia global de 10-20 casos por millón de habitantes, con una mediana de edad de presentación entre los 55 y 60 años $^{2}$. De todos estos tumores, los GIST duodenales representan menos del $5 \%$ de los casos $^{3}$.

En 1983 se establecieron las primeras características histológicas e inmunohistoquímicas que permitieron diferenciar este subgrupo de tumores. Posteriormente se descubrió la expresión del receptor tirosina
Correspondencia:

*Joel O. Jáquez-Quintana

E-mail: jomjaqui@ hotmail.com
Fecha de recepción: 31-10-2019

Fecha de aceptación: 24-05-2020 DOI: 10.24875/END.20000040
Disponible en internet: 00-00-2020

Endoscopia. 2020;32(2):69-71 www.endoscopia-ameg.com 


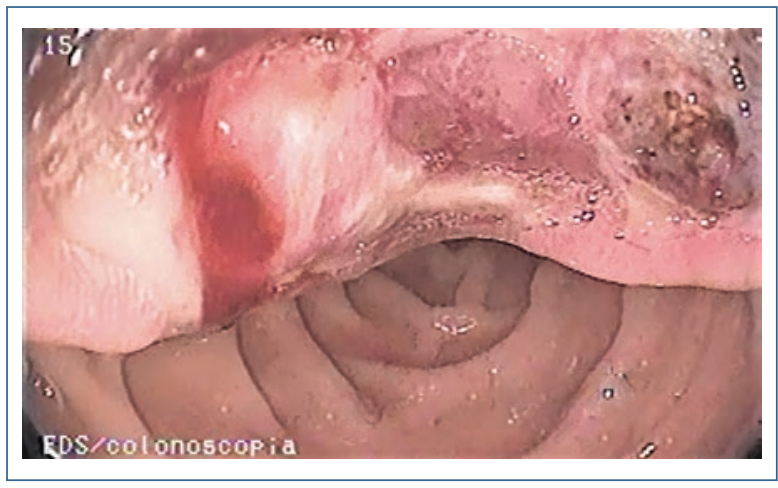

Figura 1. Lesión ulcerada de $3 \mathrm{~cm}$ en tercera porción del duodeno con bordes elevados con sangrado activo en capa y un vaso visible contralateral durante endoscopia superior.

cinasa en las células de Cajal conocido como CD117 (c-KIT) y el receptor alfa del factor de crecimiento derivado de plaquetas ${ }^{4}$. En el $90 \%$ de los GIST, las mutaciones de C-KIT dan como resultado un aumento en la actividad de la enzima tirosina cinasa, permitiendo realizar el diagnóstico al detectar esta proteína oncogénica (c-KIT) por inmunohistoquímica y un tratamiento dirigido con inhibidores de la tirosina cinasa ${ }^{5}$.

\section{Caso clínico}

Hombre de 61 años con historia de melena intermitente de ocho meses de evolución, que requirió múltiples transfusiones de concentrados eritrocitarios y uso de suplementos con hierro. Reporte de gastroscopia previa sin alteraciones, motivo por el que fue referido a nuestra institución. Se realizó endoscopia superior observando una lesión subepitelial ulcerada de aproximadamente $3 \mathrm{~cm}$ de longitud en la tercera porción del duodeno; a la inspección presentaba bordes elevados, en un extremo con un vaso visible y en el extremo contralateral con sangrado activo en capa (Fig. 1). La tomografía abdominal contrastada mostró una masa de $6.2 \times 6 \times 3.5 \mathrm{~cm}$ en la pared del duodeno entre la segunda y tercera porción sin involucro de estructuras vasculares. Al continuar con sangrado activo fue referido al departamento de cirugía, en donde se le realizó cirugía de Whipple y se inició tratamiento con imatinib. La histopatología reportó un tumor fusocelular con inmunohistoquímica CD117 positivo con índice mitótico de 2 mitosis $/ 5 \mathrm{~mm}^{2}$, concluyente para un GIST de riesgo intermedio. El paciente presenta evolución clínica favorable, en seguimiento por consulta sin presentar síntomas gastrointestinales, anemia ni datos de recurrencia o metástasis en tomografía computarizada a 12 meses de la resección quirúrgica.

\section{Discusión}

El sangrado gastrointestinal es la complicación más común, se puede observar hasta en la mitad de los casos, debido a la ulceración de la mucosa o la invasión tumoral de los vasos nutricios que causan la hemorragia ${ }^{6}$. La presentación clínica depende del tamaño y localización del tumor, que puede cursar asintomático o desarrollar síntomas inespecíficos como dolor, distensión abdominal y saciedad temprana, incluso se han reportado síntomas de obstrucción en tumores de gran tamaño $0^{1,4,5}$.

En hasta un $70 \%$ de los casos, los GIST se localizan en el estómago, del 15 al $44 \%$ en el intestino delgado", principalmente en yeyuno e íleon y un $5 \%$ en el duodeno, un $5 \%$ en el recto, menos del $1 \%$ en el esófago ${ }^{5}$ y del 2 al $11 \%$ en otras estructuras como el omento, el mesenterio o el retroperitoneo'.

Estos tumores se consideran potencialmente con riesgo de metástasis postoperatoria y recurrencia ${ }^{4}$. La edad mayor de 50 años se considera factor de mal pronóstico ${ }^{6}$, además se toma en cuenta el diámetro del tumor, el índice mitótico y el nivel de expresión del Ki67, utilizados en la escala de Fletcher modificada para categorizar el riesgo, y se utilizan mapas de contorno que estima la recurrencia postoperatoria a los 10 años ${ }^{4}$.

El tratamiento debe ser individualizado de acuerdo con el riesgo de malignidad y la presentación clínica. La resección quirúrgica se considera el estándar de oro, se recomienda la terapia sistémica adyuvante en pacientes de riesgo intermedio-alto, GIST con sangrado crónico preoperatorio y tumores grandes para prevenir la recurrencia ${ }^{4,6}$.

EI GIST duodenal debe sospecharse en cualquier paciente con una masa de pared duodenal. El crecimiento extramural y la ulceración central con o sin hemorragia gastrointestinal deben alertar al endoscopista sobre la posibilidad de este diagnóstico.

\section{Financiamiento}

La presente investigación no ha recibido ayudas específicas provenientes de agencias del sector público, sector comercial o entidades sin ánimo de lucro. 


\section{Conflicto de intereses}

Los autores declaran no tener conflicto de intereses.

\section{Responsabilidades éticas}

Protección de personas y animales. Los autores declaran que para esta investigación no se han realizado experimentos en seres humanos ni en animales.

Confidencialidad de los datos. Los autores declaran que han seguido los protocolos de su centro de trabajo sobre la publicación de datos de pacientes.

Derecho a la privacidad y consentimiento informado. Los autores han obtenido el consentimiento informado de los pacientes y/o sujetos referidos en el artículo. Este documento obra en poder del autor de correspondencia.

\section{Bibliografía}

1. El-Menyar A, Mekkodathil A, Al-Thani H. Diagnosis and management of gastrointestinal stromal tumors: An up-to-date literature review. J Cancer Res Ther. 2017;13(6):889-900.

2. Beham A, Schaefer IM, Cameron S, von Hammerstein K, Füzesi L, Ramadori G, et al. Duodenal GIST: A single center experience. Int $J$ Colorectal Dis. 2013;28(4):581-90.

3. Shen C, Chen H, Yin Y, Chen J, Han L, Zhang B, et al. Duodenal gastrointestinal stromal tumors: clinicopathological characteristics, surgery, and long-term outcome. BMC Surg. 2015;15(1):98.

4. Akahoshi K, Oya M, Koga T. Current clinical management of gastrointestinal stromal tumor. World J Gastroenterol. 2018;24(26):2806-17.

5. Miettinen M, Lasota J. Gastrointestinal stromal tumors. Gastroenterol Clin North Am. 2013;42(2):399-415.

6. Liu $Q$, Kong F, Zhou J. Management of hemorrhage in gastrointestinal stromal tumors: A review. Cancer Manag Res. 2018;10:735-43. 